\title{
Desenvolvimento e validação de um índice de vulnerabilidade social aplicado a políticas públicas do SUS
}

\author{
Development and validation of a social vulnerability index \\ applied to public policies of the Unified Health System (SUS)
}

\author{
Maria de Lourdes Drachler ${ }^{1}$ \\ Marcos Antônio de Oliveira Lobato ${ }^{2}$ \\ José Inácio Lermen ${ }^{3}$ \\ Sandra Fagundes ${ }^{3}$ \\ Alcindo Antonio Ferla ${ }^{1}$ \\ Carlos Wietzke Drachler ${ }^{4}$ \\ Luciana Barcellos Teixeira ${ }^{1}$ \\ José Carlos de Carvalho Leite ${ }^{5}$
}

\footnotetext{
${ }^{1}$ Programa de Pós-

Graduação em Saúde

Coletiva, Escola de

Enfermagem, Universidade

Federal do Rio Grande do

Sul. R. São Manoel 963, Rio

Branco. 90.620-110 Porto

Alegre RS Brasil. malu.

drachler@gmail.com

${ }^{2}$ Departamento de

Assistência Hospitalar e

Ambulatorial, Secretaria

da Saúde do Estado do Rio

Grande do Sul.

${ }^{3}$ Divisão de Atenção à

Saúde, Secretaria da Saúde

do Estado do Rio Grande

do Sul.

${ }^{4}$ Rede Governo

Colaborativo em Saúde,

Universidade Federal do Rio

Grande do Sul.

${ }^{5}$ Curso de Psicologia,

Centro Universitário La

Salle.
}

Resumo $O$ artigo apresenta o desenvolvimento e a validação inicial de um Índice de Vulnerabilidade Social - IVS-5, incluindo cinco determinantes sociais de risco à saúde, e exemplifica sua aplicação no financiamento da atenção básica pelo Sistema Único de Saúde no Rio Grande do Sul. Indicadores municipais de vulnerabilidade relativa à pobreza e dispersão populacional foram obtidos do Censo do IBGE-2010. A análise fatorial exploratória e a análise fatorial confirmatória sugerem que os cinco itens podem constituir uma escala de medida cuja confiabilidade é aceitável. O IVS-5 foi então gerado a partir do primeiro componente principal, medindo, em escores $Z$, desigualdades municipais na vulnerabilidade social relativa à pobreza e dispersão populacional no território. A validade externa do IVS-5 foi examinada em relação a desfechos de saúde, usando dados do Datasus 2007-2011, mostrando que a mortalidade infantil e as hospitalizações por condições sensíveis à atenção básica são maiores em municípios mais vulneráveis. Os resultados sugerem que o IVS-5 é medida válida de desigualdades na vulnerabilidade social entre municípios, aplicável a políticas de equidade social e em saúde.

Palavras-chave Índice de vulnerabilidade social, Alocação de recursos, Financiamento da Saúde, Equidade em Saúde, Desigualdade social 


\section{Introdução}

A distribuição de recursos para o financiamento da saúde e demais políticas sociais comprometida com a justiça social e a melhoria da qualidade de vida tem sido uma demanda populacional e um desafio para os gestores. Pobreza, definida por baixa renda, analfabetismo e baixa escolaridade, más condições de habitação e assistência inadequada à saúde descrevem as condições de vida de muitas famílias, mais frequentemente em zonas rurais e aglomerados urbanos irregulares no Rio Grande do Sul, no Brasil e internacionalmente $^{1,2}$. Tais condições inadequadas, a que essas populações estão submetidas por gerações, restringem suas opções de vida e influenciam suas práticas de saúde, expondo adultos e crianças a riscos para doenças e agravos à saúde, deficiências físicas, limitações da atividade e restrições à participação social ${ }^{3}$. Essas populações têm maior demanda de recursos e menor acesso aos serviços que necessitam ${ }^{1,4-7}$. Assim, a Política Nacional de Atenção Básica à Saúde preconiza a territorialização da atenção à saúde, com programação e execução descentralizada de ações setoriais e intersetoriais potencialmente impactantes na situação de saúde e nos condicionantes e determinantes da saúde populacional visando à equidade ${ }^{8}$. Nesse artigo assume-se a perspectiva de que o financiamento da saúde e as políticas sociais e de equidade devem voltar-se para a melhoria geral dos níveis de saúde e da oferta de serviços, com ênfase na atenção às necessidades de saúde e diminuição das desigualdades na cobertura e qualidade da atenção a grupos populacionais e segmentos da sociedade $^{1,9,10}$, conforme legislação do Sistema Único de Saúde e as principais políticas vigentes.

O financiamento equitativo da saúde previsto na legislação brasileira requer indicadores de necessidades em saúde ${ }^{1}$. Metodologias voltadas à equidade na alocação de recursos financeiros públicos em saúde no Brasil ${ }^{9-15}$ têm sido influenciadas pelo método RAWP (Resource Allocation Working Party), desenvolvido na Grã-Bretanha ${ }^{16-19}$, que estima as necessidades regionais a partir da população ponderada pela taxa nacional de utilização dos serviços, pela idade e sexo, e pela taxa de mortalidade regional padronizada.

No caso brasileiro, a implementação do financiamento equitativo da saúde torna-se complexa pelo arranjo interfederativo na saúde ${ }^{9}$, pelas transformações rápidas nos padrões sanitários, demográficos, econômicos e sociais e pela carência de análise de experiências de financiamento implementadas pelo SUS ${ }^{10}$. No Rio Grande do Sul, ex- periências de financiamento da saúde entre 1999 e 2002 foram descritas ${ }^{14,20,21}$. Desde então, o processo de cofinanciamento com recursos estaduais vem sendo aprimorado pela busca de indicadores para subsidiar os gestores da saúde, visando padrões crescentes de integralidade. Nesse sentido, o Departamento de Ações em Saúde - Divisão de Atenção Básica, da Secretaria Estadual da Saúde do Rio Grande do Sul, já incluia, na sua metodologia distributiva de recursos, vários indicadores de necessidades municipais, como o número de habitantes, a proporção de crianças e idosos na população, o inverso da renda tributária líquida per capita ${ }^{14,15,21}$, e buscava incluir também uma medida da vulnerabilidade social, indicada pela pobreza populacional e maior dificuldade na provisão dos serviços sociais e de saúde, relacionada à dispersão populacional no território. O objetivo era obter uma variável sumarizadora desse conjunto de indicadores da determinação social da saúde, visando auxiliar a distribuição dos recursos do Sistema Único de Saúde - SUS para os municípios do Rio Grande do Sul.

Este artigo descreve como foi construído e validado o Índice de Vulnerabilidade Social com os dados de municípios do Estado do Rio Grande do Sul, o qual será denominado IVS-5 por ser constituído por cinco itens. $\mathrm{O}$ artigo exemplifica a aplicação do IVS-5 pelo Governo Estadual, na Política de Incentivo Estadual à Qualificação da Atenção Básica em Saúde (PIES) e no Piso de Atenção Básica-Compensação de Especificidades Regionais. Essas políticas incluem diversas medidas articuladas a políticas federais e aumento de recursos estaduais cujos critérios distributivos devem basear-se nas necessidades populacionais e no princípio da equidade.

\section{Método}

\section{Seleção dos indicadores de vulnerabilidade social e exame da validade aparente e de conteúdo}

O objetivo era criar um indicador de vulnerabilidade social, constituído por poucos itens, obtidos de bancos de dados públicos e que representassem a relação entre a saúde e a sociedade. Seis itens indicadores de pobreza e dificuldade na provisão de serviços foram selecionados do Censo do IBGE de $2010^{22}$, para constituir os indicadores de vulnerabilidade social dos 496 municípios do Rio Grande do Sul. Os itens são: (a) percentagem de domicílios com rendimento mensal per 
capita até meio salário mínimo; (b) percentagem de domicílios não ligados à rede de distribuição de água; (c) percentagem de domicílios sem coleta de lixo; (d) percentagem de domicílios sem banheiro ou sanitário ligado à rede geral de esgoto ou pluvial; (e) percentagem de analfabetismo entre pessoas com mais de 15 anos de idade; e (f) densidade demográfica. A validade aparente e de conteúdo desses itens como indicadores de vulnerabilidade social foi examinada por profissionais da Secretaria Estadual da Saúde do Rio Grande do Sul, pela Comissão Intergestores Bipartite - CIB/RS e por especialistas da Universidade Federal do Rio Grande do Sul - UFRGS. Os cinco primeiros indicadores ('a' até 'e') são reconhecidos internacionalmente como os principais determinantes sociais de desfechos negativos na saúde populacional ${ }^{23}$. A baixa densidade populacional foi incluída como item de vulnerabilidade social, por representar uma dificuldade expressa pela CIB/RS, na provisão de atenção à saúde nos municípios com maior dispersão populacional.

Como a escala de medida diferia entre os indicadores (porcentagens e densidade demográfica), foi criada uma escala de medida comum a todos os itens. A escala de medida única foi calculada pela seguinte fórmula baseada em um índice GINI ${ }^{24}$ : Para cada indicador foi atribuído o valor 1,00 para o município em pior situação e 0,00 para o município em melhor situação. Para os demais municípios, foi calculado um valor entre zero e um usando a seguinte fórmula: diferença entre o valor do município de interesse e o valor do município em melhor situação, dividida pela diferença entre o pior e o melhor valor entre todos os municípios. Assim, para cada um dos indicadores de vulnerabilidade, foi criada uma medida de desigualdade municipal, cujos valores variaram de zero a um, sendo o valor mais alto atribuído ao município mais vulnerável e o valor mais baixo, ao menos vulnerável. Dessa forma, todos os itens da escala de vulnerabilidade social passaram a ter a mesma unidade de medida.

\section{Criação do IVS-5 e exame de sua validade interna}

O objetivo era criar um índice capaz de sumarizar desigualdades entre os municípios, considerando o conjunto dos indicadores de vulnerabilidade. Análise fatorial exploratória, análise fatorial confirmatória e exame da consistência interna do conjunto de itens foram realizados para criar o índice de vulnerabilidade social e também examinar sua validade interna, através da análise da aceitabilidade dos itens, estrutura fatorial e confiabilidade da escala ${ }^{25-27}$. Todas as análises estatísticas foram realizadas usando o pacote estatístico SPSS 17.0.

A primeira etapa da análise fatorial exploratória consistiu em examinar se a amostra dos seis indicadores de vulnerabilidade social, medidos em escala GINI, era adequada para realização de técnicas de análise fatorial. A adequação da amostra foi indicada pelo Teste Kaiser-Meyer -Olkin - KMO, esperando-se KMO maior do que 0,50 e a significância estatística do Teste de Esfericidade de Bartlett - TEB menor que $1 \%$.

Em sequência, foi examinado o desempenho de cada item para constituir uma medida sumarizadora. Os seguintes critérios de baixo desempenho foram usados: (a) baixa variabilidade em estatísticas descritivas; (b) baixa aceitabilidade, indicada pela incompletude dos dados em mais de 5\% de municípios; (c) redundância, indicada por correlação entre itens $>0,70$; (d) baixa contribuição para o total do índice de vulnerabilidade social, indicada por correlação item-total $<0,30$; (e) item que ao entrar no modelo da análise de componentes principais diminua a confiabilidade da escala, medida pelo índice de consistência interna Chronbach-alfa; e (f) quando a carga fatorial no primeiro componente principal for $<0,30$.

A confiabilidade do índice de vulnerabilidade social - como medida sumarizadora do conjunto de itens - foi indicada pelo índice de consistência interna Chronbach-alfa $\geq 0,65$ e também por índices de ajuste estimados pela técnica de análise fatorial confirmatória, que são medidas mais robustas da confiabilidade de escalas. O Chronbach-alfa é uma medida frágil da confiabilidade de escalas com poucos itens, como o índice de vulnerabilidade social desenvolvido neste estudo, pois o Chronbach-alfa tende a aumentar com o maior número de itens da escala. A eliminação de itens da escala baseou-se no desempenho métrico do item e sua relevância para a validade de conteúdo do IVS.

A análise fatorial exploratória do conjunto de itens - incluindo (a) técnica de componentes principais e (b) análise fatorial com rotações ortogonal e oblíqua da matriz de correlação - foi utilizada para identificar a estrutura fatorial mais simples e explicativa da variabilidade do conjunto de itens entre os municípios. O critério utilizado para a seleção da estrutura fatorial simples e explicativa foi o conjunto de itens que fizesse sentido teórico e que apresentasse carga $>0,40$ somente em um fator (condição verificada antes e após as rotações da matriz). A estrutura mais simples e explicativa foi encontrada no primeiro componente da análise 
de componentes principais (antes da rotação), sugerindo a possibilidade da criação de uma medida única a partir do conjunto dos itens.

A técnica de análise fatorial confirmatória foi realizada para examinar se a estrutura fatorial constituída por fator único (todos os itens do IVS formando uma única variável) se adequa aos dados dos municípios do Rio Grande do Sul. A medida da adequação foi indicada pelos seguintes índices de ajuste do modelo aos dados: significância estatística do teste qui-quadrado; qui-quadrado relativo (valor do qui-quadrado, dividido pelos graus de liberdade); erro de aproximação da raiz do quadrado médio ${ }^{28}$; índice de ajuste comparativo ${ }^{29}$; índice de ajuste normaliza$\mathrm{do}^{30}$ e índice de ajuste não normalizado (ou índice de ajuste Tucker-Lewis) ${ }^{31}$.

Como a estrutura mais simples explicativa foi encontrada no primeiro componente principal, um Índice de Vulnerabilidade Social (IVS), medido em escores $Z$, foi então gerado a partir desse componente. Isso foi feito através de uma técnica estatística para extração de variáveis, medidas em escore $Z$, a partir de componentes principais. Escalas em escore $Z$ são aquelas que apresentam distribuição normal com média igual a zero e desvio- padrão igual a um. Dessa forma, o valor do IVS municipal aqui desenvolvido indica a posição do município, em termos de vulnerabilidade social, medida em desvios-padrão acima ou abaixo da média dos 496 municípios do Estado. Valores de IVS positivos indicam que o município tem maior proporção de população vulnerável do que a média dos municípios do Estado, e valores negativos, menor proporção de população vulnerável do que a média, assim classificando os municípios em ordem crescente de vulnerabilidade.

\section{Validade Externa IVS-5}

A validade externa do IVS foi evidenciada pelas validades de construto e social. A validade de construto foi examinada investigando a relação do IVS com desfechos negativos de saúde: mortalidade infantil e hospitalização pelas 20 condições sensíveis à atenção primária, usando dados do DATA-SUS 2007-2011-RS e a lista brasileira de condições sensíveis à atenção primária ${ }^{32}$. Foram testadas as hipóteses de que a mortalidade infantil e as hospitalizações por condições sensíveis à atenção básica eram maiores nos municípios mais vulneráveis. A validade social do índice foi demonstrada pela aprovação pela CIBS-RS do uso do IVS na distribuição de recursos sociais no Rio Grande do Sul, em 2012.

\section{Resultados}

\section{Exame da validade aparente e de conteúdo dos itens do IVS-5}

Essa validade foi examinada por profissionais de Atenção Básica em Saúde e membros da $\mathrm{CIB} / \mathrm{RS}$ consideraram que a prevalência de baixa renda familiar, analfabetismo, ausência de água encanada, falta de esgotamento sanitário e ausência de coleta de lixo no domicílio são indicadores de pobreza populacional e determinantes sociais de desfechos negativos na saúde, e que a baixa densidade populacional dificulta a provisão de atenção à saúde. Assim, eles concluíram que esses itens são suficientes para identificar desigualdades municipais na vulnerabilidade social relacionada à pobreza e à dificuldade na provisão de serviços devido à dispersão populacional no território.

\section{Análise estatística dos itens do IVS-5}

Dados sobre os seis indicadores estavam disponíveis para todos os municípios do Estado, sugerindo que a cobertura dos itens é excelente. A Tabela 1 apresenta a ocorrência dos indicadores de vulnerabilidade e o índice GINI de desigualdade municipal, para cada indicador de vulnerabilidade social relacionado à pobreza e à dispersão populacional no território, Rio Grande do Sul, IBGE 2010. No índice GINI, para cada indicador, foi calculado tendo como referência municípios em menor vulnerabilidade. Os valores mínimo e máximo de cada indicador evidenciam grande variação na vulnerabilidade social entre municípios do Estado.

A correlação entre os indicadores de vulnerabilidade social medida em escala GINI foi examinada. Observou-se redundância entre o índice GINI da renda familiar per capita menor do que meio salário mínimo, e o índice GINI para analfabetismo em maiores de 15 anos (Coeficiente de correlação de Pearson $=0,79$ ), havendo necessidade de exclusão de um desses itens da escala. A opção foi pela exclusão do analfabetismo, porque a renda familiar apresentou maior contribuição para a escala do IVS, indicada por maior correlação item-total e maior peso fatorial. Os cinco itens restantes não apresentaram redundância (média da correlação interítens de 0,09 a 0,57) e parecem contribuir para a escala do IVS (todos os cinco itens com correlação item-total $>0,33$ ). Por constituir-se de cinco itens, o índice criado foi denominado IVS-5. 


\section{Identificação da estrutura fatorial e avaliação das propriedades métricas do IVS-5}

A análise fatorial exploratória dos cinco itens mantidos no IVS-5 evidenciou cargas fatoriais altas $(>0,55)$ no primeiro componente principal e no primeiro fator da matriz após rotação ortogonal, sugerindo que os cinco índices GINI de desigualdades municipais são bons indicadores da variável latente IVS (Tabela 2). O quadrado da carga fatorial ( $r^{2}$ ou coeficiente de correlação múltipla ao quadrado) estima a percentagem da variância daqueles índices GINI que é explicada pelo IVS. Assim, o IVS-5, gerado a partir do primeiro componente principal, explica $67,2 \%$ da variância entre os municípios na ausência de coleta de lixo $\left(0,82^{2}\right) ; 46,2 \%$ da variância da renda familiar per capita $<0,5$ salários mínimos $\left(0,68^{2}\right)$; $41 \%$ da variância da falta de esgotamento sanitário $\left(0,64^{2}\right)$; e $30 \%$ da variância da ausência de água tratada encanada $\left(0,55^{2}\right)$ e da variância na densidade demográfica $\left(0,55^{2}\right)$. O IVS-5 explicou em média $43 \%$ da variância desses itens entre os municípios. A Consistência Interna, medida pelo índice alfa de Cronbach foi 0,66, sugerindo que a confiabilidade do IVS-5 é aceitável (Tabela 2). A análise fatorial confirmatória evidenciou que os cinco itens organizados em fator único apresentam ajuste adequado aos dados municipais do Rio Grande do Sul (Tabela 3).

\section{Validade externa do IVS-5}

O IVS-5 municipal variou de $-4,28$ a 2,55 , com média igual a zero e desvio padrão igual a
1,00. O IVS-5 ordena os municípios em ordem crescente de vulnerabilidade social. Valores de IVS-5 positivos indicam maior proporção de população em situação de vulnerabilidade, e valores negativos, menor proporção de população em vulnerabilidade, comparados à média de IVS-5 de todos os municípios do Estado.

A Secretaria Técnica da CIB/RS aprovou a utilização do IVS-5, para informar duas políticas de cofinanciamento equitativo da atenção básica a saúde pela Secretaria Estadual da Saúde do Rio Grande do Sul em 2012. Especificamente:

a) O IVS-5 foi o critério de seleção de municípios para alocação de recursos da Política de Atenção Básica-Compensação de Especificidades Regionais - PAB-CER, que em 2012, totalizou R\$ 9.823.276,80, quantia suficiente para prover $\mathrm{R} \$$ 15,00 per capita a uma população de até 655.000 habitantes. Os municípios foram ordenados em

Tabela 2. Estrutura fatorial exploratória dos índices GINI de desiguldade municipal e medida de confiabilidade do IVS-5.

\begin{tabular}{lc}
\hline \multicolumn{1}{c}{ Itens } & $\begin{array}{c}\text { Carga fatorial } \\
\text { no primeiro } \\
\text { componente } \\
\text { principal }\end{array}$ \\
\hline 1. Ausência de coleta de lixo & 0,82 \\
2. Renda familiar per capita $<0,5$ & 0,68 \\
salário mínimo & \\
3. Falta de esgotamento sanitário & 0,64 \\
4. Ausência de água tratada encanada & 0,55 \\
5. Densidade demográfica & 0,55 \\
Confiabilidade & Cronbach- $\alpha=0,66$
\end{tabular}

Tabela 1. Ocorrência dos indicadores de vulnerabilidade social e índice GINI de desigualdade municipal nesses indicadores, Rio Grande do Sul, 2010.

\begin{tabular}{|c|c|c|c|c|c|c|c|c|c|c|c|c|}
\hline & \multicolumn{2}{|c|}{$\begin{array}{c}\text { Famílias } \\
\text { com renda } \\
\text { per capita } \\
<0,5 \text { salário } \\
\text { mínimo } \\
\end{array}$} & \multicolumn{2}{|c|}{$\begin{array}{c}\text { Analfabetismo } \\
\text { em }>\text { de } 15 \\
\text { anos } \\
\end{array}$} & \multicolumn{2}{|c|}{$\begin{array}{l}\text { Domicílios } \\
\text { sem água da } \\
\text { rede pública }\end{array}$} & \multicolumn{2}{|c|}{$\begin{array}{l}\text { Domicílios } \\
\text { sem sanitário } \\
\text { em rede de } \\
\text { esgoto ou } \\
\text { pluvial } \\
\end{array}$} & \multicolumn{2}{|c|}{$\begin{array}{c}\text { Domicílios } \\
\text { sem coleta de } \\
\text { lixo } \\
\end{array}$} & \multicolumn{2}{|c|}{$\begin{array}{c}\text { Densidade } \\
\text { demográfica }\end{array}$} \\
\hline & $\%$ & Gini & $\%$ & Gini & $\%$ & Gini & $\%$ & Gini & $\%$ & Gini & $\begin{array}{c}\text { Habitantes } \\
\qquad / \mathrm{Km}^{2}\end{array}$ & Gini \\
\hline Mínimo & 2,9 & 0,00 & 1,0 & 0,00 & 0,7 & 0,00 & 14,1 & 0,00 & 0,1 & 0,00 & 1,6 & 0,00 \\
\hline Máximo & 56,8 & 1,00 & 20,1 & 1,00 & 98,9 & 1,00 & 100,0 & 1,00 & 79,6 & 1,00 & $2.932,0$ & 1,00 \\
\hline Percentil 25 & 13,9 & 0,20 & 4,1 & 0,17 & 11.0 & 0,11 & 74,4 & 0,70 & 5,2 & 0,64 & 13,6 & 0,986 \\
\hline Percentil 50 & 22,3 & 0,36 & 6,4 & 0,28 & 22,0 & 0,23 & 95,7 & 0,95 & 18,3 & 0,23 & 23,3 & 0,993 \\
\hline Percentil 75 & 30,6 & 0,51 & 8,9 & 0,41 & 37,8 & 0,38 & 99,3 & 0,99 & 36,8 & 0,46 & 42,7 & 0,996 \\
\hline
\end{tabular}

Fonte dos dados: Censo do IBGE $2010^{22}$ 
ordem decrescente de vulnerabilidade social até que esse número de habitantes foi alcançado pelos 126 municípios mais vulneráveis do Estado (IVS-5 municipal >0,63). A Tabela 4 mostra que os municípios selecionados para receber recursos do PAB-CER tinham menos de 100.000 habitantes e apresentavam maior proporção de hospitalizações por condições sensíveis à atenção básica, maior taxa de hospitalizações por essas condições e maior coeficiente de mortalidade infantil do que os municípios menos vulneráveis, sejam eles municípios com mais de 100.000 habitantes ou municípios menos populosos.

b) O IVS-5 foi incluído a partir de 2012 entre os critérios na distribuição equitativa dos recursos da Política de Incentivo Estadual à Qualificação da Atenção Básica em Saúde (PIES). A Tabela 5 apresenta distribuição do repasse da PIES, conforme faixas do Índice de vulnerabilidade social - IVS-5.

Tabela 3. Índices de ajuste do IVS na análise fatorial confirmatória.

\begin{tabular}{|c|c|c|}
\hline \multirow[b]{2}{*}{ Nome (sigla) } & \multicolumn{2}{|c|}{ Índices de Ajuste } \\
\hline & Valor desejável & Valor estimado \\
\hline Qui-quadrado $(\chi 2)$, significância estatística. & $\chi 2, p \geq 0,05$ & $2.160(\mathrm{p}=0,340)$ \\
\hline Qui-quadrado relativo $\left(\chi^{2} / \mathrm{gl}\right)^{*}$ & $<2$ & 1,08 \\
\hline Erro de aproximação da raiz do quadrado médio & $<0,08$ & 0,01 \\
\hline Índice de ajuste comparativo & $>0,95$ & 0,999 \\
\hline Índice de ajuste normalizado & $>0,95$ & 0,999 \\
\hline Índice de ajuste não-normalizado ${ }^{* *}$ & $>0,95$ & 0,999 \\
\hline
\end{tabular}

"Qui-quadrado relativo = Qui-quadrado/graus de liberdade, ${ }^{* *}$ Também conhecido como Índice de ajuste de Tucker-Lewis.

Tabela 4. Ocorrência de eventos de saúde e população de municípios selecionados e não selecionados para receber recursos da Política de Atenção Básica de Compensação de Especificidades Regionais (PAB-CER), Rio Grande do Sul, 2012.

\begin{tabular}{|c|c|c|c|}
\hline \multirow{2}{*}{ Ocorrência de eventos de saúde } & \multirow{2}{*}{$\begin{array}{c}\text { Selecionados }^{*} \\
<100.000 \\
\text { habitantes }\end{array}$} & \multicolumn{2}{|c|}{ Não selecionados } \\
\hline & & $\begin{array}{r}<100.000 \\
\text { habitantes }\end{array}$ & $\begin{array}{r}\geq 100.000 \\
\text { habitantes }\end{array}$ \\
\hline \multicolumn{4}{|c|}{$\begin{array}{l}\text { Internações por condições sensíveis à atenção primária, } \\
2008 \text { a } 2010\end{array}$} \\
\hline Percentagem do total de hospitalizações & 32,64 & 27,06 & 19,01 \\
\hline Taxa por 1000 habitantes & 23,83 & 18,99 & 12,38 \\
\hline \multicolumn{4}{|l|}{ Mortalidade infantil, 2005 a 2010} \\
\hline Coeficiente por 1000 nascidos vivos & 14,32 & 12,25 & 12,49 \\
\hline População total & 660.885 & 5.032 .373 & 5.000 .962 \\
\hline Número de municípios & 126 & 352 & 18 \\
\hline
\end{tabular}

"Selecionados = Índice de Vulnerabilidade Social > 0,63 desvios-padrão da média.

Tabela 5. Repasse da Política de Incentivo Estadual à Qualificação da Atenção Básica em Saúde-PIES em 2012, conforme o Índice de Vulnerabilidade Social Municipal.

\begin{tabular}{|c|c|c|c|}
\hline \multirow[b]{2}{*}{$\begin{array}{l}\text { Índice de Vulnerabilidade Social } \\
\text { (desvios-padrão da média) }\end{array}$} & \multicolumn{3}{|c|}{ Repasse do PIES } \\
\hline & $\begin{array}{l}\text { Municípios } \\
\text { favorecidos }\end{array}$ & $\begin{array}{l}\text { População } \\
\text { favorecida }\end{array}$ & $\begin{array}{c}\text { Valor per } \\
\text { capita (Reais) }\end{array}$ \\
\hline$<-0,50$ & 149 & 8.200 .996 & 0,85 \\
\hline$-0,50$ a 0.00 & 99 & 1.105 .957 & 1,73 \\
\hline 0,01 a 1,00 & 171 & 994.915 & 4,00 \\
\hline$>1,00$ & 76 & 392.061 & 8,00 \\
\hline Total & 496 & 10.693 .929 & 1,50 \\
\hline
\end{tabular}

"Selecionados = Índice de Vulnerabilidade Social > 0,63 desvios-padrão da média. 


\section{Discussão}

O objetivo deste artigo foi descrever o desenvolvimento do índice de vulnerabilidade social (IVS-5) e sua validação inicial para os municípios do Rio Grande do Sul, exemplificando sua aplicação pela Secretaria de Estado da Saúde (SES/RS) na Política de Incentivo Estadual à Qualificação da Atenção Básica em Saúde (PIES) e no Piso de Atenção Básica-Compensação de Especificidades Regionais (PAB-CER), que incluem diversas medidas articuladas a políticas federais e aumento de recursos com critérios distributivos baseados nas necessidades populacionais e no princípio da equidade, cujo embasamento teórico originou-se na década de 70, na Grã Bretanha, pelo trabalho do grupo RAWP-Resource Allocation Working Party.

O IVS-5 foi criado como uma medida comparativa da vulnerabilidade social entre municípios, relacionada à pobreza da população e à dificuldade em prover serviços sociais e de saúde a populações com baixa densidade populacional. Dessa forma, o IVS-5 é uma medida relativa, pois mensura as desigualdades entre os municípios em termos de vulnerabilidade, e não constitui uma medida de nível absoluto de vulnerabilidade. O desenvolvimento do IVS-5 visou auxiliar os gestores estaduais de saúde a estabelecerem critérios de alocação de recursos financeiros, materiais e humanos em diferentes áreas do território baseados no princípio de equidade, cumprindo as disposições legais que normatizam o funcionamento do SUS.

A criação do IVS-5 envolveu equipes técnicas da SES/RS, especialistas do campo da Saúde Coletiva e gestores e técnicos dos sistemas municipais e regionais de saúde, além de representantes do controle social e, portanto, mobilizou a aprendizagem para a gestão equitativa, como um primeiro resultado obtido. A validade aparente e de conteúdo dos itens como indicadores de vulnerabilidade social relacionada à pobreza e à baixa densidade populacional foi examinada por profissionais da Secretaria Estadual da Saúde, por especialistas da Universidade Federal do Rio Grande do Sul - UFRGS e pela CIB/RS. Os cinco indicadores que compõe o IVS- 5 podem ser facilmente obtidos do site do IBGE, censo populacional de 2010. A escolha dos indicadores de vulnerabilidade baseou-se na determinação social da saúde, na facilidade de interpretação dos indicadores e na disponibilidade de dados confiáveis para todos os municípios em site de domínio público. A exclusão do item medindo prevalência de analfabetismo não parece ter afe- tado a validade de conteúdo do IVS-5, porque o item analfabetismo mostrou-se redundante, pela alta correlação com a baixa renda familiar per capita, um dos desfechos da baixa escolaridade no Brasil. O conteúdo do IVS-5 foi validado em instâncias de controle social no Estado (CIB/RS).

O exame da validade interna do IVS-5 inclui análise exploratória de componentes principais e análise fatorial exploratória, complementadas por análise fatorial confirmatória. As correlações moderadas entre cada item e o total do IVS-5 mostram que os cinco itens contribuem para a medida do IVS-5. O índice de consistência interna aceitável (Chronbach- $=0,66)$ sugere que os cinco itens constituem uma escala de medida confiável. Na análise fatorial confirmatória, os índices de ajuste do modelo, em que os cinco itens constituem um índice único - o IVS-5, sugerem que esse modelo é adequado aos dados censitários municipais do Rio Grande do Sul, em 2010 (Tabela 3). Tais índices de ajuste têm sido considerados indicadores robustos da validade de variáveis latentes ${ }^{33}$, como o IVS-5.

O IVS-5 explica cerca de $43 \%$ da variância dos cinco indicadores de vulnerabilidade social entre os municípios (soma das cargas fatoriais ao quadrado na Tabela 2, dividido por cinco). Por ser um índice padronizado medido em escores $Z$, o IVS-5 permite identificar a posição do município em desvios-padrão acima ou abaixo da média, referente à vulnerabilidade social devido à pobreza e a dispersão populacional no território. Para avaliações de desigualdades populacionais, o escore- $Z$ é considerado mais adequado do que outras medidas, como percentis, quartis ou percentagens da mediana, porque as diferenças em torno da média são semelhantes, facilitando comparações entre os grupos populacionais ${ }^{34,35}$.

A criação do IVS-5 permitiu mostrar a grande variação na vulnerabilidade social entre os municípios do Rio Grande do Sul, pois a amplitude de variação do IVS-5 foi de 6,83 desvios-padrão entre o município menos vulnerável (IVS-5 $=-4,28)$ e o mais vulnerável (IVS- $5=2,55$ ).

A validade externa do IVS-5 foi sugerida por sua relação com a ocorrência de desfechos negativos de saúde no município. Por exemplo, os municípios mais vulneráveis apresentaram maiores taxas de hospitalização por condições sensíveis à atenção básica e maior coeficiente de mortalidade infantil, do que os municípios menos vulneráveis, sugerindo que o IVS-5 pode indicar municípios com maiores necessidades em saúde.

Instancias de controle social no Rio Grande do Sul aprovaram o uso do IVS-5 para informar 
políticas de cofinanciamento equitativo da atenção básica a saúde a partir de 2012. Assim, o IVS5 tem sido o único critério da alocação de recursos da PAB-CER e um dos critérios da distribuição equitativa de recursos da PIES (juntamente com o tamanho da população, o inverso da renda tributaria líquida per capita e a proporção de idosos e crianças, já utilizados em anos anteriores a 2012). Esses recursos financeiros visam o custeio de ações e serviços de atenção básica, registrados nos Planos de Saúde Municipais vigentes e aprovados pelos Conselhos Municipais de Saúde. Esses Planos baseiam-se em prioridades informadas por indicadores de saúde pactuados, visando a melhoria da saúde populacional e a redução de internações hospitalares por condições sensíveis à atenção básica. Para receber os recursos do PAB-CER, o município deve apresentar um plano de utilização dos recursos na atenção básica à saúde e também um plano de atuação intersetorial para minimizar a vulnerabilidade social relativa à pobreza no município, sendo um incentivo à mudança nessa situação de saúde.
Esses resultados sugerem que o IVS-5 é uma medida concisa, confiável e válida das desigualdades entre os municípios, na vulnerabilidade relacionada à pobreza e à menor densidade populacional, potencialmente útil para instrumentalizar o cofinanciamento equitativo da atenção básica à saúde no Rio Grande do Sul. De modo semelhante o IVS-5 poderá ser utilizado na alocação de recursos para qualificação da atenção de média e alta complexidade em saúde e para informar políticas e programas setoriais ou intersetoriais envolvendo também as seguintes áreas: cultura, educação, habitação e saneamento, obras públicas, irrigação e desenvolvimento urbano, segurança pública, justiça e direitos humanos, trabalho, turismo, esporte e lazer, planejamento, gestão e participação cidadã, ciência, inovação e desenvolvimento tecnológico. O IVS-5 poderá ser ampliado para incluir outros indicadores sociais (constituindo IVS-n), conforme a realidade e necessidades de cada região. Futuros estudos devem analisar a validade do IVS-5 para o país, outros estados brasileiros e distritos ou bairros das grandes metrópoles.

\section{Colaboradores}

ML Drachler, MAO Lobato, JI Lermen, S Fagundes e AA Ferla participaram da concepção do índice de vulnerabilidade social; ML Drachler, JI Lermen, CW Drachler, LB Teixeira e JCC Leite realizaram a análise de dados. Todos os autores participaram da redação do artigo. 


\section{Referências}

1. Drachler ML, Côrtes SMV, Castro JD, Leite JCC. Proposta de metodologia para selecionar indicadores de desigualdade em saúde visando definir prioridades de políticas públicas no Brasil. Cien Saude Colet 2003; 8(2):461-470.

2. Comissão para os Determinantes Sociais da Saúde (OMS). Redução das desigualdades no período de uma geração: Igualdade na saúde através da ação sobre os seus determinantes sociais. Relatório Final da Comissão para os Determinantes Sociais da Saúde. Lisboa: Organização Mundial da Saúde; 2010. [acessado 2012 maio 24]. Disponível em: http://whqlibdoc.who.int/publications /2010/9789248563706_por.pdf

3. Organização Mundial da Saúde (OMS). International Classification of Functioning, Disability and Health-ICF; 2003. [acessado 2012 maio 24]. Disponível em: http:// www3.who.int/icf/icftemplate.cfm?myurl=introduction. html\%20\&mytitle=introduction.

4. Schmidtlein MC, Deutsch RC, Piegorsch WW, Cutter SL. A Sensitivity Analysis of the Social Vulnerability Index. Risk Analysis 2008; 28(4).

5. Freitas PF, Drachler ML, Leite JCDC, Marshall T. Inequalities in cesarean delivery rates by ethnicity and hospital accessibility in Brazil. Int J Gynecol Obstet 2009; 107(3):198-201.

6. Drachler ML, Leite JCDC, Hooper L, Hong CS, Pheby D, Nacul L, Lacerda E, Campion P, Killett A, McArthur $\mathrm{M}$, Poland F. The self-expressed needs of people with Chronic Fatigue Syndrome/Myalgic Encephalomyelitis: A systematic review. BMC Public Health (Online) 2009; 9:458. [acessado 2012 maio 24]. Disponível em: http://www.biomedcentral.com/1471-2458/9/458.

7. Drachler MLD, Marshall TM, Aerts DRGC, Leite JCC, Freitas PFFF. Housing infrastructure modifies the effect of social inequalities on child development: A multilevel analysis. Eur J Epidemiology 2006; 21(Supl. S):147-147.

8. Brasil. Ministério da Saúde (MS). Secretaria de Atenção à Saúde. Departamento de Atenção Básica. Política $\mathrm{Na}$ cional de Atenção Básica. Brasília: MS; 2012.

9. Ribeiro P. Distribuição de recursos financeiros e equidade: uma relação delicada na saúde. Cien Saude Colet 2005; 10(2):292-296.

10. Mendes Á, Leite MG, Marques RM. Discutindo uma Metodologia para a Alocação Equitativa de Recursos Federais para o Sistema Único de Saúde. Saúde e Sociedade 2011; 20(3):673-690.

11. Castro JD. Análise da distribuição dos recursos financeiros para a assistência à saúde no Brasil e no Rio Grande do Sul - Proposta de operacionalização dos critérios para a sua distribuição. Saúde em Debate 1991; 32(1):10-16.

12. Piola SF. O financiamento da descentralização dos serviços de saúde: critérios para a transferência de recursos federais para estados e municípios. Brasília: OPAS, WHO; 1990. (Série Economia e Financiamento 1).

13. Ferreira MP, Dini NP, Ferreira SP. Espaços e dimensões da pobreza nos municípios do estado de São Paulo. Índice Paulista de Vulnerabilidade Social (IPVS). São Paulo em Perspec 2006; 20(1):5-17.

14. Pelegrini MLM, Castro JD, Drachler ML. Equidade na alocação de recursos para a Saúde: a experiência do Rio Grande do Sul, Brasil. Cien Saude Colet 2005; 10(2):275286.
15. Brasil. Política de Incentivo Estadual à Qualificação da Atenção Básica em Saúde (PIES). Porto Alegre: Secretaria Estadual da Saúde, Governo do Estado do RGS; 2011. [acessado 2012 maio 24]. Disponível em: http:// www.saude.rs.gov.br/conteudo/521/?Pol\%C3\%ADtica de_Incentivo_Estadual_\%C3\%A0_Aten\%C3\%A7\%C3\%A3o_B\%C3\%A1sica.

16. Department of Health and Social Security. Sharing resources for health in England. Report of the resources allocation working party. Londres: DHSS; 1976.

17. Department of Health and Social Security. Review of the RAWP formula. Final report by the NHS Mangement Board. Londres: DHSS; 1988.

18. Foster DP. Social class differences in sickness and general practitioner consultations. Health Trends 1976; 8(4):18-34.

19. Ham C. Health policy in Britain. The politics and organization of national health sector. Hong Kong: Mac Millan; 1992.

20. Ferla AA, Fagundes SMS. Tempo de inovações: a experiência da gestão na saúde do Rio Grande do Sul. Porto Alegre: Editora Da Casa; 2002.

21. Ferla AA, Fagundes SMS. O fazer em Saúde Coletiva: inovações da atenção à saúde o Rio Grande do Sul. Porto Alegre: Editora Da Casa; 2002.

22. Instituto Brasileiro de Geografia e Estatística (IBGE). Censo Demográfico 2010; Rio de Janeiro: IBGE; 2011. [acessado 2012 maio 24]. Disponível em: http:// www.censo2010.ibge.gov.br/.

23. Comissão Nacional sobre Determinantes Sociais da Saúde (CNDSS). As causas das iniquidades em saúde no Brasil. Relatório final. 2008. [acessado 2013 jul 13]. Disponível em: http://bvsms.saude.gov.br/bvs/publicacoes/causas_sociais_iniquidades.pdf.

24. Instituto de pesquisa econômica aplicada (IPEA). Índice de Gini. [acessado 2013 jul 13]. Disponível em: http://www.ipea.gov.br.

25. Hair JR, Joseph F. Análise multivariada de dados. $6^{\mathrm{a}} \mathrm{ed}$. Porto Alegre: Bookman; 2009.

26. Tabachnick BG, Fidell LS. Using Multivariate Statistics. $4^{\text {th }}$ ed. Boston: Allyn \& Bacon; 2001.

27. Velicer WF, Jackson DN. Component analysis versus common factor analysis: some further observations. Multivar Behav Res 1990; 25(1):97-114.

28. Browne MW, Cudeck R. Alternative ways of assessing model fit. In: Bollen KA, Long JS, editors. Testing structural equation models. Newsbury Park: Sage; 1993. p. 136-162.

29. Bollen KA. Structural equations with latent variables. New York: Wiley; 1989.

30. Bentler PM, Bonnet DG. Significance tests and goodness of fit in the analysis of covariance structures. Psychological Bulletin 1980; 88(3):588-606.

31. Sharma S, Mukherjee S, Kumar A, Dillon WR. A simulation study to investigate the use of cutoff values for assessing model fit in covariance structure models. $J$ Bus Res 2005; 58(1):935-943.

32. Alfradique ME, Bonolo PF, Dourado I, Lima-Costa MF, Macinko J, Mendonça CS, Oliveira VB, Sampaio LFR, De Simoni C, Turci MA. Internações por condições sensíveis à atenção primária: a construção da lista brasileira como ferramenta para medir o desempenho do sistema de saúde (Projeto ICSAP - Brasil). Cad Saude Publica 2009; 25(6):1337-1349. 
33. Pett MA, Nancy RL, Sullivan JJ. Making sense of factor analysis: The use of factor analysis for instrument development in health care research. Thousand Oaks: Sage Publications; 2003.

34. Erreygers G. Beyond the health concentration index: an Atkinson alternative to the measurement of socioeconomic inequality of health. Antwerpen: University of Antwerpen; 2006. [acessado 2012 maio 24]. Disponível em: http://www.bvsde.paho.org/bvsacd/cd56/erreygers -170806.pdf

35. Bambas L, Braveman P, Dachs JNW, Delgado I, Gakidou E, Moser K, Rolfe L, Vega J, Zarowsky C. Strengthening health information systems to address health equity challenges. B World Health Organ 2005; 83(8):561-640.

Artigo apresentado em 17/07/2013

Aprovado em 31/10/2013

Versão final apresentada em 11/11/2013 